Technical
Memorandum

"THIS MEMORANDUM IS NOT TO BE REPRODUCED WITHOUT THE EXPRESS WRITTEN PERMISSION OF SANDIA CORPORATION AND THE AUTHOR"

$$
\text { 174-54-51 }
$$

THE RESPONSE CHARACTERISTICS OF ATRPLANE AND MISSILE PRESSURE MEASURING SYSTEMS

\title{
Harold Vaughn
}

\section{ABSTRACT}

An empirical method for determining the response characteristics of pressure measuring systems used in aircraft and missiles is presented. Both experimental and theoretical treatments are included. The method is more rigorous than the ordinary linear treatment usually used in the analysis of aircraft systems. The method is presented in such a manner as to be easily applicable to most pressure measuring systems with greater than critical damping. 


\section{DISCLAIMER}

This report was prepared as an account of work sponsored by an agency of the United States Government. Neither the United States Government nor any agency Thereof, nor any of their employees, makes any warranty, express or implied, or assumes any legal liability or responsibility for the accuracy, completeness, or usefulness of any information, apparatus, product, or process disclosed, or represents that its use would not infringe privately owned rights. Reference herein to any specific commercial product, process, or service by trade name, trademark, manufacturer, or otherwise does not necessarily constitute or imply its endorsement, recommendation, or favoring by the United States Government or any agency thereof. The views and opinions of authors expressed herein do not necessarily state or reflect those of the United States Government or any agency thereof. 


\section{DISCLAIMER}

Portions of this document may be illegible in electronic image products. Images are produced from the best available original document. 


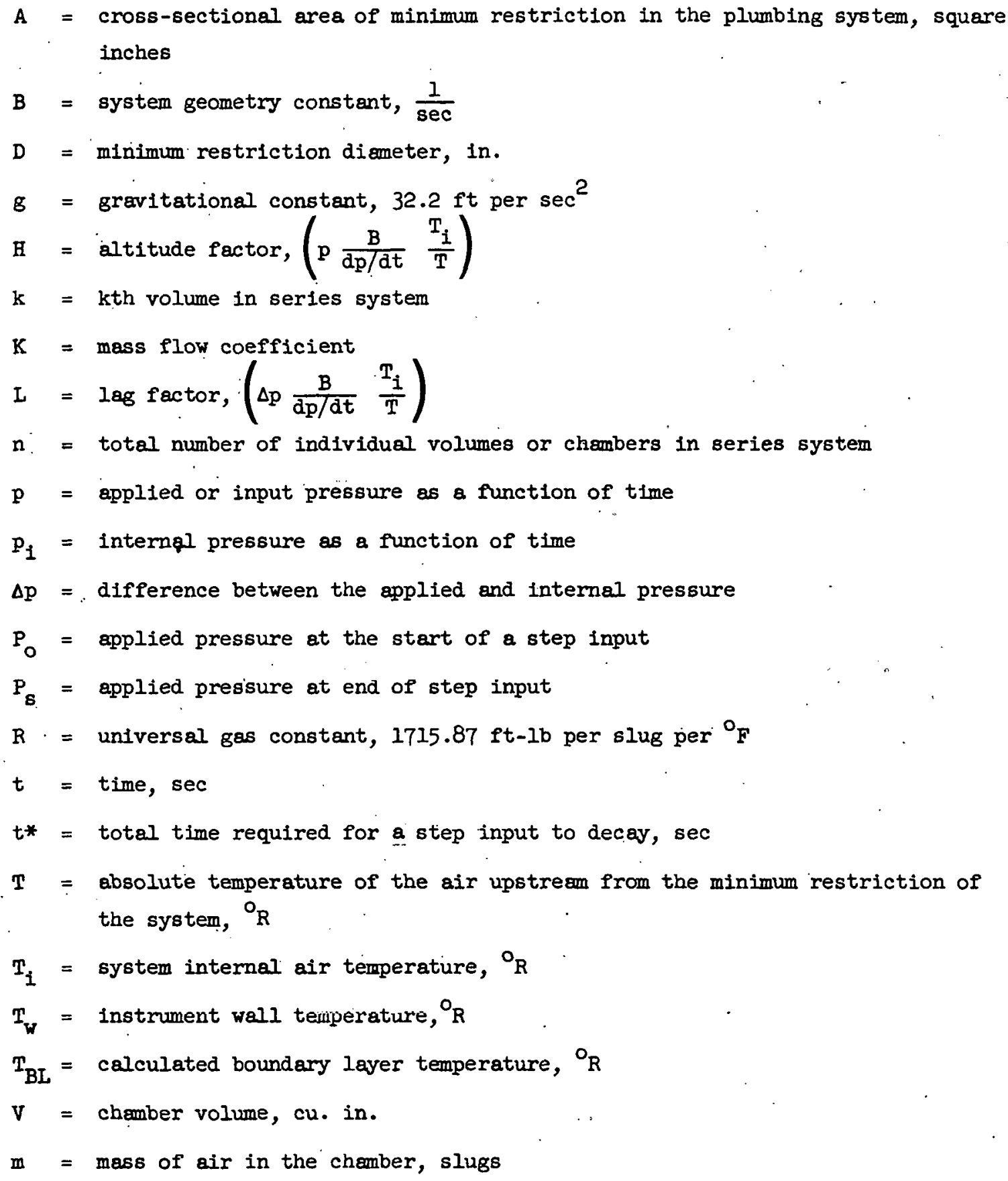




\section{INTRODUCTION}

The rapid increase in velocities of test vehicles, airplanes, and missiles during the last few years has brought about the necessity of being able to predict the response, or more conmonly the lag, experienced by the pressure measuring instrumentation in these vehicles during diving, climbing or accelerating maneuvers at high speeds. The pressure lag in some instances causes a large percentage error in the measured pressure; consequently, the response of the instrumentation cannot be ignored as being within the accuracy of the instrumentation.

For several years a linear treatment of the problem has been discussed in a number of papers. $1,2,3$ This method makes use of a linear differential equation which describes the response of a resistance-capacity electrical system. By intuitive reasoning this equation is transformed into a pressure equation where the electrical resistance is a function of viscosity, diameter of plumbing and length of plumbing leading to the chamber representing the measuring instrument, and the electrical capacity is represented as a function of the internal pressure and the volume of the instrument. The solution to this equation makes it possible to determine a time constant from an experimental test of the system where a step input in pressure is applied. This time constant is usually multiplied by the applied rate of change of pressure to arrive at the lag in pressure. A number of experimental step pressure input tests were made at Sandia Laboratory on various systems of interest with the idea of using the linear analysis and possibly extending it to accommodate more complicated series- and parallel-connected instruments. However, it immediately beçame apparent that the time constant determined in this manner was dependent on the size of the pressure step that was applied. This inconsistency was recognized by some authors 4 and it was suggested that the time constant be determined for a number of step sizes so that the data could be extrapolated to a zero step size, since the inconsistency was thought to be the result of varying the Reynolds number of the flow for different step sizes. To the author, however, the obvious conclusion was that regardless of the cause, the linear equation could not be used if the time constant was not in actuality a constant. This led to the development of the quasi-empirical theory shown below. By using this theory, which is nonlinear, it was possible to show that the linear time constant actually approaches zero as the step size approaches zero; consequently, it is believed that the application of a linear differential equation to the problem of pressure lag in pressure measuring systems can lead to very large errors. 
ANALYSIS OF THE BASIC SYSTEM.

The development of this nonlinear theory is based on an empirical equation which describes the mass flow through a sharp-edged orifice in terms of the pressure ratio across the orifice. This empirical relation was developed by Perry. 5 Early attempts to use other mass flow equations were unsuccessful, becamse they were unable to predict the flow through the rough interior of normal pneumatic plumbing. The equation derived by Perry is

$$
\frac{d \dot{m}}{d t}=\frac{0.465 A}{g \sqrt{T}} \sqrt{P_{1}^{2}-P_{2}^{2}}
$$

where $P_{1}$ and $P_{2}$ are respectively the steady state upstream and downstream pressures across the orifice and $T$ is the upstream temperature. The pressures in Eq. (1) can be time-dependent, if it is assumed that the delay in response of the mass flow to changes in pressure differential are insignificant. This, of course, means that it is assumed that the effect of the inertia of the air and the effect of the speed of pressure propagation in the plumbing is accountable. ${ }^{1}$ This is believed to be a reasonable assumption as long as the plumbing lines are not extremely long. Then, with a change in symbollsm, Eq. (1) becomes:

$$
\frac{d m}{d t}=\frac{K A}{g \sqrt{T}} \sqrt{\left|p^{2}-p_{i}^{2}\right|}
$$

where $K$ is a constant for a particular type of plumbing and a given Reynolds number. The $\mathrm{K}$ is essentially a constant over a falrly. wide range of Reynolds number. Figure 1 shows a schematic of the basic system under consideration, which consists of an orifice opening into a chamber. The orifice represents the minimum restriction area present in the entire plumbing system, and the chamber represents a pressure measuring instrument such as that found in an aptopilot or altimeter. It should be noted that any effect of the length of plumbing lines is considered to be absorbed into the constant $K$ as far as Reynolds number is concerned, and any additional volume contributed by the lines can be added to the chamber volume. If the plumbing lines are very long, the system should be treated as a series system where the volume associated with the plumbing is treated as a separate chamber. Now, the equation of state of the air inside the chamber is,

$$
p_{1}=\frac{m R T_{1}}{V}
$$


Remembering that $p_{1}$ is a function of time, the derivative of Eq. (3) is:

$$
\frac{d p_{i}}{d t}=\frac{R T_{i}}{V} \frac{d m}{d t}+m \frac{R}{V} \frac{d T_{i}}{d t}
$$

Fortunately, the last term of Eq. (4) is normally insignificant with respect to the flrst term. This nas recently been shown by flight data where $T_{i}$ changed approximately $12^{\circ} \mathrm{F}$ in a time interval of four minutes while the incoming air temperature changed some $340^{\circ} \mathrm{F}$. This can be explained by the fact that pressure measuring instruments with enough internal volume to cause a significant lag in pressure response have such a large internal surface area and such a large mass with respect to the internal air mess, that the instrument acts as an efficient heat sink or source. The result is that the internal air temperature follows the instrument wall temperature very closely. Since the instrument temperature is relatively constant in most installations, it follows that the time derivative of $T_{i}$ is approximately zero. Then

$$
\frac{d p_{i}}{d t}=\frac{R T_{i}}{v} \frac{d m}{d t}
$$

Thus, Eqs. (2) and (5) can be combined to yield,

$$
\frac{d p_{1}}{d t}=B \frac{T_{1}}{T} \sqrt{\left|p^{2}-p_{1}^{2}\right|}
$$

Where

$$
B=\frac{K R}{B} \quad \frac{A}{V} \sqrt{T}
$$

and $\mathbf{T}$ is the appropriate upstream temperature. The system constant $B$ is essentially a constant for a given Reynolds number regime and upstream temperature. Equation (6) is the basic differential equation relating the response of the internal pressure $p_{1}$ to the applied pressure $p$, and can be solved for any time dependent pressure input by computing machines, such as the R.E.A.C. It can also be solved for a step pressure input, which is useful in determining the system constant $B$. Finally, it can be solved explicitly for an input pressure that varies linearly with time, which is similar to the input applied to an instrument installed in an airplane or missile that is either diving or climbing. The solution to Eq. (6) 
for a step pressure input that goes from $P_{0}$ to $P_{s}$ at $t=0$ where $P_{0}<P_{s}$ and $T_{i}=T$, is:

$$
p_{i}=P_{s} \sin \left(B t+\sin ^{-1} \frac{P_{0}}{P_{s}}\right), P_{0}<P_{s}
$$

and Eq. (8) can be written in terms of a unit dimensionless step, as

$$
\frac{P_{s}-P_{i}}{P_{s}-P_{0}}=\frac{P_{s}}{P_{s}-P_{0}}\left[1-\sin \left(B t+\sin ^{-1} \frac{P_{0}}{P_{s}}\right)\right], P_{0}<P_{s}
$$

Note that step pressure input may be in the form of either an increasing or a decreasing step, however the increasing pressure step was used on all experimental tests of this paper.

The companion equations for $\mathrm{P}_{\mathrm{o}}>\mathrm{P}_{\mathrm{S}}$ are:

$$
\begin{gathered}
P_{i}=\frac{\left(P_{0}+\sqrt{P_{0}^{2}-P_{s}^{2}}\right)}{2} e^{-B t}+\frac{P_{s}^{2}}{2\left(P_{0}+\sqrt{P_{0}^{2}-P_{s}^{2}}\right)} e^{B t}, P_{0}>P_{s} \\
\frac{p_{i}-P_{s}}{P_{0}-P_{s}}=\frac{1}{P_{0}-P_{s}} \cdot\left[\frac{\left(P_{0}+\sqrt{P_{0}^{2}-P_{s}^{2}}\right)}{2} e^{-B t}+\frac{P_{s}^{2}}{2\left(P_{0}+\sqrt{P_{0}^{2}-P_{s}^{2}}\right)} e^{B t}\right], P_{0}>P_{s}
\end{gathered}
$$

Now, Eqs. (8) and (10) provide a very convenient method for determining the system constant $B$, since they can be solved for $B$ when $t *$ is the total time to decay $(t=t *)$. These equations are:

$$
\mathrm{B}=\frac{\pi / 2-\sin ^{-1} P_{\mathrm{O}} / \mathrm{P}_{\mathrm{B}}}{\mathrm{t}^{*}}, \mathrm{P}_{\mathrm{O}}<\mathrm{P}_{\mathrm{S}}
$$

and

$$
B=\frac{1}{t^{*}} \ln \left(\frac{P_{0}+\sqrt{P_{0}^{2}-P_{s}^{2}}}{P_{s}}\right), P_{0}>P_{s}
$$


The two sets of Eqs. (9) and (11), (12) and (13), are the two methods of determining B from experïmental data. In applying Eqs. (9) and (11), the method is essentially a trial and error curve fitting process. Equations (12) and (13) are used by simply determining from the experimental data, the time required for the response to a step input to decay. The $B^{\prime} s$ determined from these two methods differ by about 12 per cent in the case of a step pressure input of $25 \mathrm{~mm}$. This is demonstrated on Fig. 2, where the experimental response to a step pressure input is represented by the symbols; the solid. line representing Eq. (9) for a B of 0.70 and the dotted line respresenting Eq. (9) for a B of 0.62 determined from $\mathrm{Eq}$. (12). It can be seen that the theory for $B=0.70$ agrees well with the experimental response to the step but begins to diverge toward the end, while the theory for $B=0.62$, determined from Eq. (12), disagrees by about 12 per cent over the first part of the step response, but agrees with the end. It is believed that this is caused partially by the Reynolds number of the flow through the plumbing varying from about 100,000 at the start to zero at the end of the response to the step. This could cause the mass flow coefficient $K$ to change from one value at the start of the step to a smaller value as the Reynolds number decreased toward the end of the step. Of course, this would be consistent with the change in the $B$ during the step response: In general, the Reynolds number of the flow through the plumbing during flight is on the order of a few thousand, consequently, the $B$ determined by Eq. (12) where $B$ is dependent on the total time for the response to a step input to decay, yields better results for flight applications. This is indicated by the dotted line in Fig. 2 .

In order to show the dependence of the system constant B on the ratio of minimum restriction area to the volume of the chember a number of experimental tests were run on various basic systems, as shown in Fig. 1 , with different $D^{2} / v$ ratios. The volumes of the test systems varied from 23.5 cubic inches to 92 cubic inches and the restrictions were AN fittings with dimeters ranging from 0.125 inch to 0.172 inch of both straight and elbow types. The connecting plumbing was all AN standard pneumatic plumbing. The B's were then determined from Eq. (12) and plotted versus $D^{2} / V$ in Fig. 3. The test results are plotted in Fig. 3 and it can be seen that $B$ is linearly dependent on the $D^{2} / v$ ratio of the system. If no experimental data can be obtained, the $B$ can be calculated by

$$
B=7500 \frac{D^{2}}{V} \text { (for AN straight fittings) }
$$


and

$$
B=4750 \frac{D^{2}}{V} \text { (for AN elbow fittings) }
$$

where $D$ is in inches and $V$ in cublc inches.

Caution should be exercised in the application of Eqs. (14) and (15) since they are applicable only in the case of systems with short lines with considerably larger internal diameters than the dimeter of the fittings. The experimental method of determining $B$ must be used if the system is not of this nature.

A solution to Eq. (6) can be obtained where p, the input pressure, varies linearly with time. This solution makes it possible to apply the theory to flight problems, since the rate of change of pressure during a dive or climb can be approximated as a straight line. Since,

$$
p=\frac{d p}{d t} t+P_{c}
$$

where

$$
p=P_{c} \text { at } t=0
$$

The lag in pressure is,

$$
\Delta p=p-p_{i}=\frac{d p}{d t} t-p_{i}+P_{c}
$$

Substituting $\mathrm{Eq} .(16)$ in (6) yields

$$
\frac{d p}{d t}-\frac{d \Delta p}{d t}=\frac{T_{1}}{T} B \sqrt{2 p \Delta p-(\Delta p)^{2}}
$$

An epproximate solution to Eq. (17) that is accurate enough for most cases can be obtained by assuming $\mathrm{d} \Delta \mathrm{p} / \mathrm{dt}$ small compared to $\mathrm{dp} / \mathrm{dt}$ and $(\Delta \mathrm{p})^{2}$ small compared to $2 p \Delta p$. The resulting equation is

$$
\Delta p=\left(\frac{T}{T_{i}}\right)^{2}\left(\frac{d p}{d t}\right)^{2} \frac{1}{2 B^{2} p}
$$

The exact solution can be obtained by substituting the lag factor,

$$
I=\Delta p \frac{B}{d p / d t} \frac{T_{1}}{T}
$$


and the altitude factor

$$
\mathrm{H}=\mathrm{p} \frac{\mathrm{B}}{\mathrm{dp} / \mathrm{dt}} \frac{\mathrm{T}_{\mathrm{i}}}{\mathrm{T}}
$$

in Eq. (17) which produces the normalized equation,

$$
\frac{\mathrm{dL}}{\mathrm{dH}}=1-\sqrt{L(2 \mathrm{H}-\mathrm{L})} \text {. }
$$

Equation (21) can be solved by an iterative process. This solution has been performed and plotted on Fig. 4. Consequently, if $B, d p / d t, p$ and $T_{i} / T$ are known, L can be found from Fig. 4 and $\Delta p$ easily calculated. Writing Eq. (18) in terms of $\mathrm{L}$ and $\mathrm{H}$ yields,

$$
L=\frac{1}{2 \mathrm{H}}
$$

which can be used for $\mathrm{H} \geq 5$.

In order to check the validity of the theory, a test was performed on a system, the step response of which was shown in Fig. 2, in which the input pressure varied at an approximately constant rate. The lag in pressure in the system was measured with a small Statham pressure transducer. The variation of input pressure, rate of change of input pressure, measured and theoretical pressure lag as a function of time are shown in Fig. 5. It is felt that the agreement between the theoretical and experimental $\Delta p$ is adequate, particularly when the difficulty of measuring such a small transient pressure is considered. It can be seen on Fig. 5 that the theory does not predict the response during the first four seconds as accurately as it does during the last thirteen seconds. This probably is due to the high Reynolds number (about 25,000) existing at the start of the test.

For the convenience of the reader, Eq. (18) is rewritten below in terms of the temperatures that exist in flight, for the conditions of ascent and descent through the atmosphere.

Ascent:

$$
\Delta p=-\frac{T_{0}}{T_{w}}\left(\frac{d p}{d t}\right)^{2} \frac{1}{2 B_{0}^{2} p}
$$

Descent:

$$
\Delta p=\frac{T_{0} T_{B L}}{T_{w}^{2}}\left(\frac{d p}{d t}\right)^{2} \frac{1}{2 B_{O}^{2} p}
$$


where $B_{0}$ is the system constant determined in the laboratory at a free air temperature of $T_{O}$, and $T_{B L}$ and $T_{w}$ are the calculated boundary layer temperatures at the inlet to the plumbing system and the instrument wall temperature respectively in flight.

The solution for a linear input discussed above can be used to calculate the response to any input as long as $\mathrm{d}^{2} \mathrm{p} / \mathrm{dt}^{2}$ is small. If the input is such that $\mathrm{d}^{2} \mathrm{p} / \mathrm{dt^{2 }}$ is large, then the solution to Eq. (6) for the particular input must be obtained by a computer such as R.E.A.C.

\section{SERIES SYSTEMS}

Fortunately, the theory can be applied to complex systems where the chambers are connected in either a series or parallel manner. A schematic diagram is shown in Fig. 6 with the symbolism used in the derivation. A system of three chambers is chosen for analysis since its solution leads to a mathematical pattern which allows a general solution to be written. Starting with the third chamber and using the same assumptions that were used in the analysis of the basic system, it can be seen that

$$
\mathrm{p}_{3}=\frac{\mathrm{m}_{3}}{\mathrm{v}_{3}} \cdot R \mathrm{RT}_{\mathrm{i}}
$$

and

$$
\frac{d p_{3}}{d t}=\frac{d m_{3}}{d t} \frac{R T_{1}}{V_{3}}
$$

From Eq. (2)

$$
\frac{d m_{3}}{d t}=\frac{k_{3} A_{3}}{g \sqrt{T_{i}}} \sqrt{\left|p_{2}^{2}-p_{3}^{2}\right|}
$$

Substituting Eq. (27) in (26) yields

$$
\frac{d p_{3}}{d t}=B_{3} \sqrt{\left|p_{2}^{2}-p_{3}^{2}\right|}
$$


where

$$
B_{3}=\frac{K_{3} A_{3}}{V_{3}} \frac{R \sqrt{T_{1}}}{B}
$$

A similar line of ressoning can be followed in deriving

$$
\frac{d p_{2}}{d t}=B_{2} \sqrt{\left|p_{1}^{2}-p_{2}^{2}\right|}-\frac{v_{3}}{v_{2}} B_{3} \sqrt{\left|p_{2}^{2}-p_{3}^{2}\right|}
$$

and

$$
\frac{d p_{1}}{d t}=\frac{T_{i}}{T} \quad B_{1} \sqrt{\left|p^{2}-p_{1}^{2}\right|}-\frac{V_{2}}{V_{1}} B_{2} \sqrt{\left|p_{1}^{2}-p_{2}^{2}\right|}
$$

where

$$
B_{2}=\frac{K_{2} A_{2}}{V_{2}} \cdot \frac{R \sqrt{T_{i}}}{B}
$$

and

$$
B_{1}=\frac{K_{1} A_{1}}{V_{1}} \times \frac{\sqrt{T}}{B}
$$

An approximate solution for an input with constant dp/dt can be obtained for Eqs. (28), (30) and (31) by using a similar treatment to that used in the derivation of Eq. (18). This solution is,

$$
\begin{gathered}
p-p_{1}=\Delta p_{k=1}=\left(\frac{d p}{d t}\right)^{2} \frac{1}{2 p}\left[\left(\frac{T}{T_{I}} \frac{1}{B_{1}}+\frac{1}{B_{2}}+\frac{1}{B_{3}}\right)^{2}\right] \\
p-p_{2}=\Delta p_{k=2}=\left(\frac{d p}{d t}\right)^{2} \frac{1}{2 p}\left[\left(\frac{T}{T_{i}} \frac{1}{B_{1}}+\frac{1}{B_{2}}+\frac{1}{B_{3}}\right)^{2}+\left(\frac{1}{B_{2}}+\frac{1}{B_{3}}\right)^{2}\right] \\
p-p_{3}=\Delta p_{k=3}=\left(\frac{d p}{d t}\right)^{2} \frac{1}{d t}\left[\left(\frac{T}{T_{i}} \frac{1}{B_{1}}+\frac{1}{B_{2}}+\frac{1}{B_{3}}\right)^{2}+\left(\frac{1}{B_{2}}+\frac{1}{B_{3}}\right)^{2}+\left(\frac{1}{B_{3}}\right)^{2}\right]
\end{gathered}
$$

Equations (34), (35) and (36) can be written into a general equation expressing the lag of the kth chamber with respect to the input to the complete system for 
a system of $\mathrm{n}$ chambers. That 1 ,

$$
\begin{aligned}
\Delta \mathrm{p}_{k}=\left(\frac{\mathrm{dp}}{\mathrm{dt}}\right)^{2} \frac{1}{2 \mathrm{p}} & {\left[\left(\frac{\mathrm{T}}{\mathrm{T}_{1}} \frac{1}{\mathrm{~B}_{1}}+\frac{1}{\mathrm{~B}_{2}}+\cdots \frac{1}{\mathrm{~B}_{\mathrm{n}}}\right)^{2}+\left(\frac{1}{\mathrm{~B}_{2}}+\frac{1}{\mathrm{~B}_{3}}+\cdots+\frac{1}{\mathrm{~B}_{\mathrm{n}}}\right)^{2}\right.} \\
& \left.+\cdots+\left(\frac{1}{\mathrm{~B}_{\mathrm{k}}}+\cdots+\frac{1}{\mathrm{~B}_{\mathrm{n}}}\right)^{2}+\delta_{k n} \frac{1}{\mathrm{~B}_{\mathrm{n}}^{2}}\right]
\end{aligned}
$$

where

$$
\delta_{k n}\left\{\begin{array}{l}
=1 \text { if } k=n \\
=0 \text { if } k \neq n
\end{array}\right.
$$

It can be seen that Eq. (37) will reduce to Eq. (18) for $\mathrm{n}=1$. This leads to the interesting conclusion that Fig. 4 is applicable to series systems as long as $(1 / B)^{2}$ in Eq. (18) is replaced by the bracketed term in Eq. (37). It should be noted that $B_{1}, B_{2} \ldots B_{n}$ are the system constants determined for each individual inlet-volume combination in the system. The B's should be determined from the experimental step response obtained from each chamber, with its associated plumbing, when it is completely disconnected from the system. Lack of space prevents the presentation of experimental data that exists on series systems, although these data corroborate the derivation shown above.

\section{PARALIEL SYSTEMS}

The parallel system does not yield to theoretical analysis until some basic observations are made regarding the nature of the operation of the system. Figure 7 is a schematic diagram of the system where the A's are in all cases the minimum restriction area of the plumbing lines. Following the mass flow concept, it can be seen that if

$$
A \leq A_{1}+A_{2},
$$

then $A$ becomes the controlling entrance and both chambers will respond in a similar manner and the $B$ of the total system is a function of $A /\left(V_{1}+v_{p}\right)$. In this case, the response of both chambers is identical and the system $B$ can be determined experimentally by the some methods as used for the basic system. The only other flow condition exists when

$$
A>A_{1}+A_{2}
$$


and in this case the tro chambers respond individually in the same manner as they would if completely separated. Then $B_{1}$ is a function of $A_{1} / V_{1}$ and $B_{2}$ is a function of $\mathrm{A}_{2} / \mathrm{V}_{2}$. The $\mathrm{B}^{\prime} \mathrm{s}$ are determined experimentally by recording the response of each chamber to a step input.

\section{CONCLUSION}

The theory provides a rigorous method for the analysis of simple and complex pressure measuring systems, which has been substantiated in the laboratory and in - Plight. The effects of instrument temperature and boundary layer temperature on the pressure lag is shown, and has been partially substantiated in flight at high Mach numbers. A particularly useful aspect of the theory, that has been treated only lightly in the text, is that the basic differential equation can be solved for any arbitrary pressure input by computing machines, as long as the acoustical lag is not significant.

While more experimental testing is needed to determine accurately the variation of the system constent with Reynolds number and different types of plumbing, this method of analysis is presented with the belief that it is highly useful in predicting the response, in flight, of highly damped pressure measuring $6 y s t e m s$.

DISTRIBUTION:

W. H. Lawrence, 1923-2 (6)

R. F. Brodsky, 5141 (2)

G. Byrne, 1922-2 (2)

Case No. 409.03

Aupust 23, 1954 
1. Huston, Wilbur B., "Accuracy of Airspeed Measurements, and Flight Calibration Procedure", NACA TN No. 1605, 1948.

2. Wildhack, W. A., "Pressure Drop in Tubing in A1rcraft Instrument Installat1ons", NACA TN No. 593, 1937.

3. Dello, G. J., Schwent, G. V., and Cesaro, R. S., "Transient Behavior of Lumped-Constant Systems for Sensing Gas Pressures", NACA TN No. 1988, 1949.

4. Head, Richard M., "Lag Determination of Altimeter Systems", Journal of the Aeronautical Sciences, Vol. 12, No. 1, pp. 85-93, 1945.

5. Perry, J. A., "Critical Flow Through Sharp-Edged Orifices", Transactions of the ASME, October 1949. 


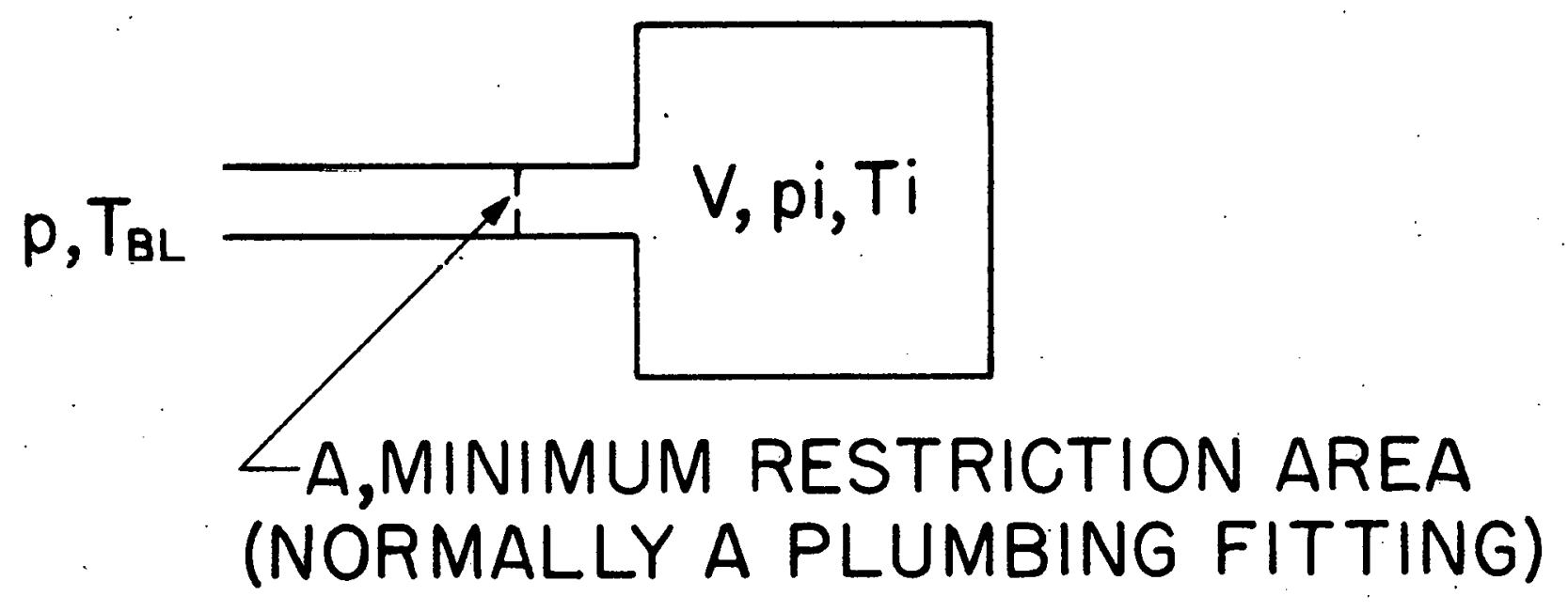

FIG.I SCHEMATIC DIAGRAM OF BASIC SINGLE CHAMBER SYSTEM 


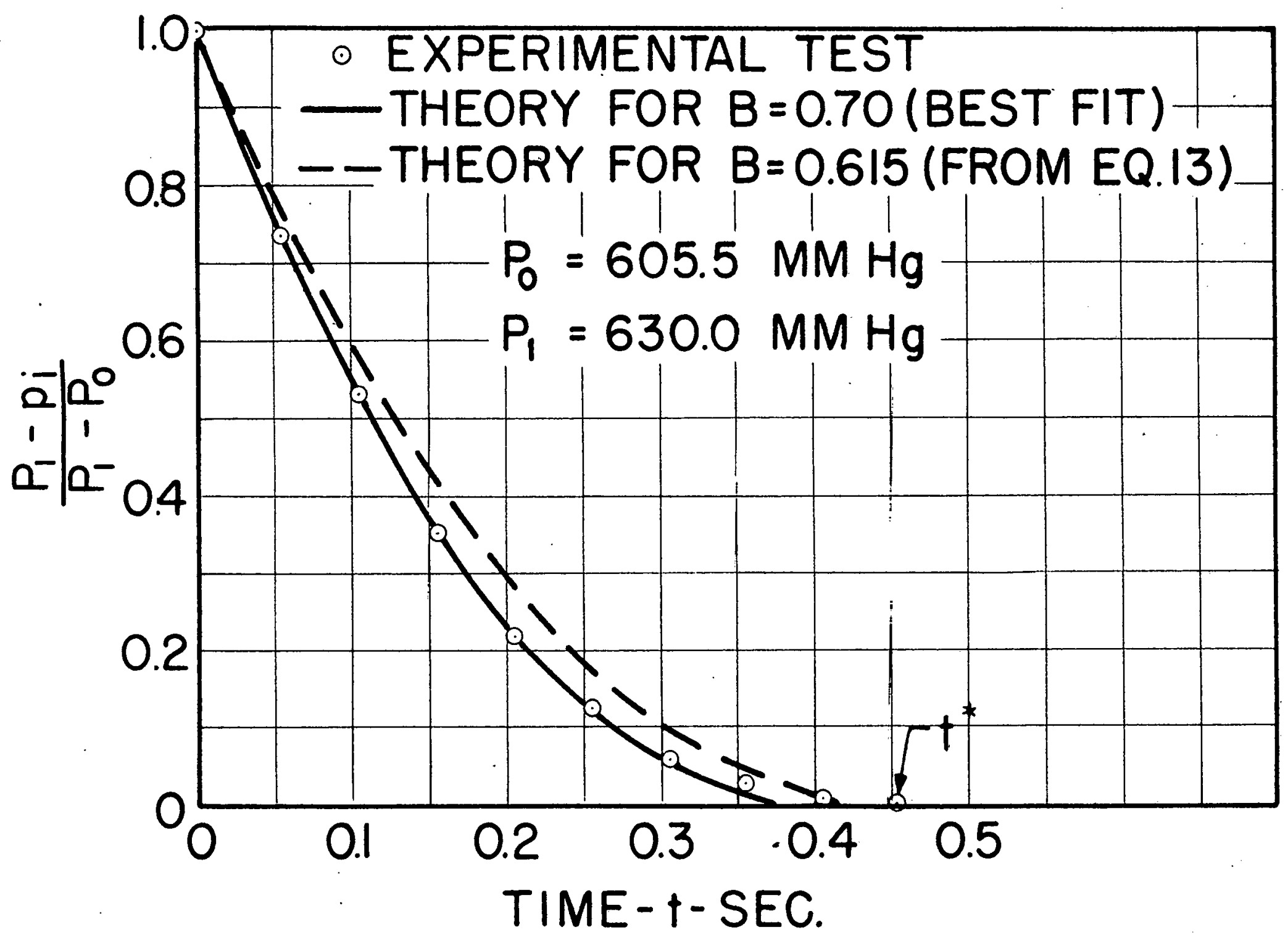

FIG. 2 COMPARISON OF THEORY WITH EXPERIMENT FOR STEP PRESSURE INPUT TO SYSTEM 


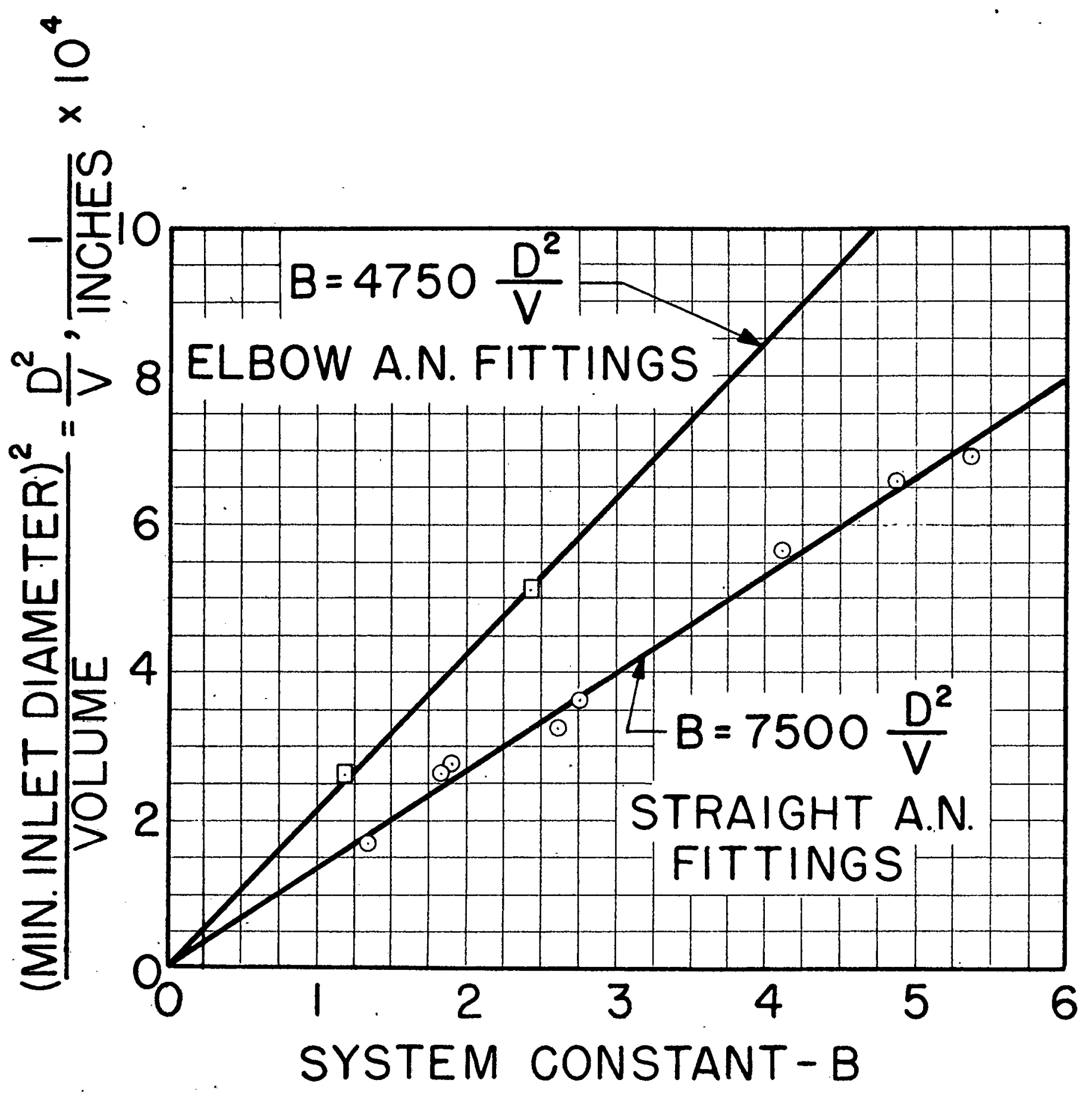

FIG 3 SYSTEM CONSTANT FOR VARIOUS MINIMUM RESTRICTION DIAMETER TO CHAMBER VOLUME RATIOS 


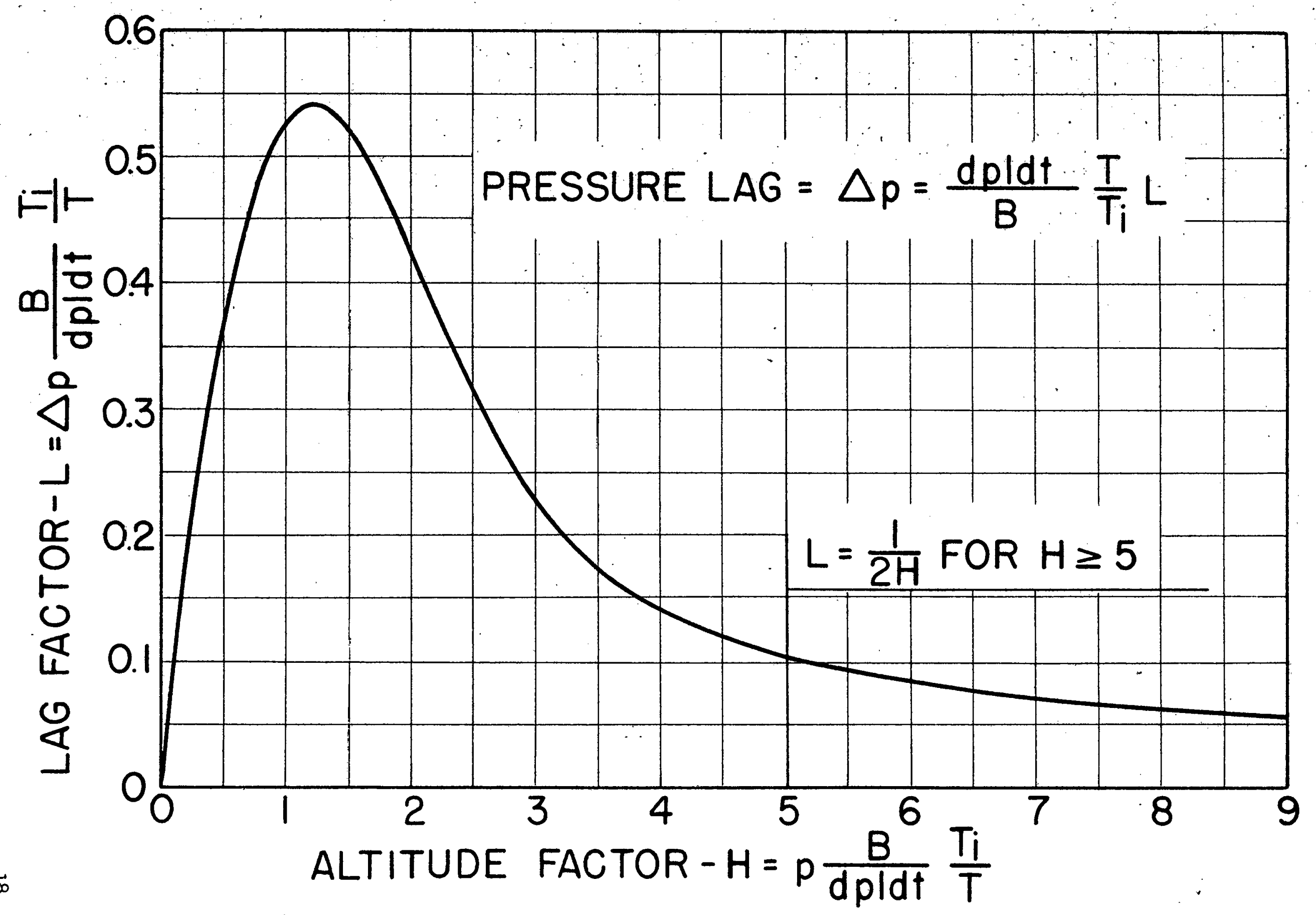

FIG. 4 LAG FACTOR AS A FUNCTION OF ALTITUDE FACTOR 


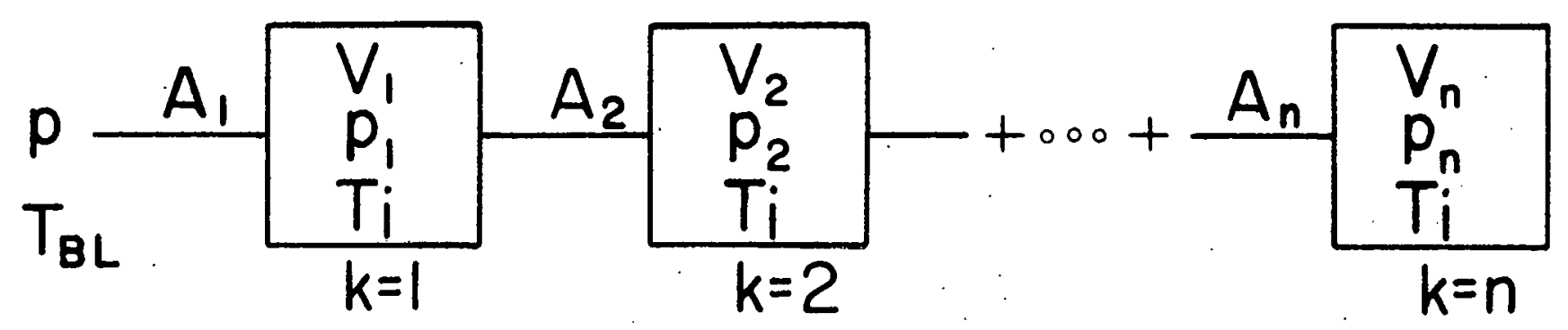

FIG. 6 SERIES SYSTEM SCHEMATIC DIAGRAM 


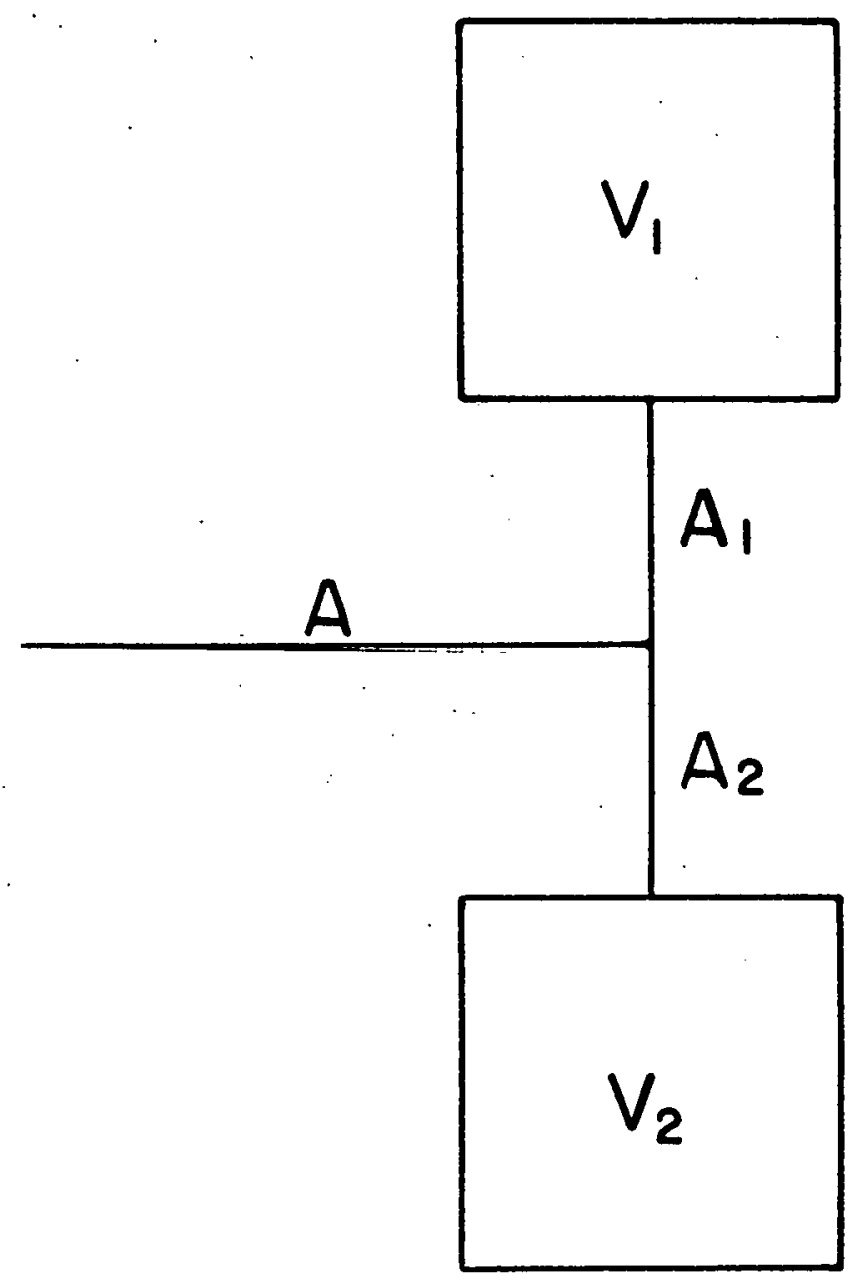

FIG. 7 SCHEMATIC DIAGRAM OF THE PARALLEL SYSTEM 